\title{
QUADRICS ASSOCIATED WITH A CURVE ON A SURFACE
}

\author{
V. G. GROVE
}

1. Introduction. Many of the important contributions to projective differential geometry of non-ruled surfaces are concerned with systems of quadrics associated with a point and a curve on the surface. Many of these quadrics belong to a certain family, a characterization of which is the main purpose of this paper.

Let the homogeneous projective coordinates $\left(x^{1}, x^{2}, x^{3}, x^{4}\right)$ of a general point $x$ on a non-ruled surface $S$ be given as functions of the asymptotic parameters $u, v$, and let these functions be so normalized that they satisfy the Fubini canonical system of differential equations,

$$
\begin{aligned}
x_{u u} & =\theta_{u} x_{u}+\beta x_{v}+p x, \\
x_{v v} & =\gamma x_{u}+\theta_{v} x_{v}+q x, \quad \theta=\log (\beta \gamma),
\end{aligned}
$$

wherein the coefficients satisfy certain integrability conditions [7]. ${ }^{1}$ The abbreviations

$$
\phi=\partial \log \left(\beta \gamma^{2}\right) / \partial u, \quad \psi=\partial \log \left(\beta^{2} \gamma\right) / \partial v
$$

will be found useful.

Let $C_{\lambda}$, a curve on $S$ through $x$, be considered as imbedded in a one-parameter family of curves defined by the differential equation

$$
d v-\lambda d u=0 .
$$

Since the homogeneous coordinates of any point $X$ may be written in the form

$$
X=x_{1} x+x_{2} x_{u}+x_{3} x_{v}+x_{4} x_{u v},
$$

the coordinates of $X$ referred to the tetrahedron $x, x_{u}, x_{v}, x_{u v}$ may be taken as $\left(x_{1}, x_{2}, x_{3}, x_{4}\right)$.

It is remarkable that many of the equations of quadrics associated with $S$ and $C_{\lambda}$ at $x$ are of the form

$$
x_{2} x_{3}+T x_{4}=0
$$

wherein

$$
T=-x_{1}+k_{2} x_{2}+k_{3} x_{8}+k_{4} x_{4}
$$

and

Received by the editors August 24, 1944.

1 Numbers in brackets refer to the references cited at the end of the paper. 


$$
k_{2}=l_{2} \beta / \lambda+m_{2} \gamma \lambda^{2}, \quad k_{3}=l_{3} \beta / \lambda^{2}+m_{3} \gamma \lambda,
$$

$l_{2}, m_{2}, l_{3}, m_{3}$ being constants and $k_{4}$ a parameter. In particular the quadrics of Darboux, of Moutard, of Davis [3], all of the quadrics derived by $\mathrm{Wu}[9]$, the conjugal quadrics [5], the asymptotic osculating quadrics, and the quadrics Hsiung [6] has associated with $C_{\lambda}$ at $x$ all belong to the system (1). We shall denote this family by $Q\left(l_{2}, m_{2}, l_{3}, m_{3}\right)$.

2. A characterization of the family. Let a line $l$ be determined by the points $\rho \sigma$ with coordinates given by the expressions $\rho=x_{u}-b x$, $\sigma=x_{v}-a x$. The $R_{\lambda}$-associate of $l$, as defined by Bell [1], joins the points whose coordinates are

$$
\rho_{\lambda}=\rho+\beta x / \lambda, \quad \sigma_{\lambda}=\sigma+\gamma \lambda x .
$$

Bell [1] has called the one-parameter family of curves defined by

$$
d v-\mu d u=0, \quad \mu=-\beta /\left(\gamma \lambda^{2}\right)
$$

the $R_{\lambda}$-derived curves, and has characterized them in terms of the $R_{\lambda}$-associate of $l$. The $R_{\mu}$-associate of $l$ joins the points

$$
\rho_{\mu}=\rho-\gamma \lambda^{2} x, \quad \sigma_{\mu}=\sigma-\beta x / \lambda^{2} .
$$

From (4) and (5) we easily prove the following theorems.

The $R_{\lambda}$-associate of $l$ coincides with the $R_{\mu}$-associate of $l$ if and only if $C_{\lambda}$ is a curve of Darboux.

The $R_{\lambda}$-associate of $l, l$, and the $R_{\mu}$-associate of $l$ intersect the asymptotic tangents in points which with $x$ are harmonic if and only if $C_{\lambda}$ is a curve of Segre.

Now define points $R, S, R_{\lambda}, S_{\lambda}$ by the cross ratio equations

$$
\begin{aligned}
& \left(x, \rho_{\lambda}, \rho_{\mu}, R\right)=k, \quad\left(x, R, \rho, R_{\lambda}\right)=K, \\
& \left(x, \sigma_{\lambda}, \sigma_{\mu}, S\right)=l, \quad\left(x, S, \sigma, S_{\lambda}\right)=L,
\end{aligned}
$$

$k, l, K, L$ being constants. One finds readily that the coordinates of $R_{\lambda}, S_{\lambda}$ are given by the expressions

$$
R_{\lambda}=\rho+k_{2} x, \quad S_{\lambda}=\sigma+k_{3} x
$$

wherein $k_{2}, k_{3}$ are given by (3) and

$$
\begin{array}{llrl}
l_{2} & =k K, & & m_{2}=K(k-1), \\
l_{3}=L(l-1), & & m_{3}=l L .
\end{array}
$$

We shall call the line joining $R_{\lambda}, S_{\lambda}$ the $S_{\lambda}\left(l_{2}, m_{2}, l_{3}, m_{3}\right)$-associate of $l$. 
The reciprocal $l^{\prime}$ of the line $l$ joins $x$ to the point with coordinates $x_{u v}-a x_{u}-b x_{v}$. It is easy to show that the most general quadric having second order contact with $S$ at $x$ and with respect to which the polar line of $l^{\prime}$ is the $S_{\lambda}\left(l_{2}, m_{2}, l_{3}, m_{3}\right)$-associate of $l$ is the quadric $Q\left(l_{2}, m_{2}, l_{3}, m_{3}\right)$.

3. The general transformation of Cech. The coordinates of any point $R$ on the tangent to $C_{\lambda}$ at $x$ may be written in the form

$$
R=x_{u}+\lambda x_{v}+t x .
$$

The polar plane of $R$ with respect to $Q\left(l_{2}, m_{2}, l_{3}, m_{3}\right)$ has coordinates $\left(u_{1}, u_{2}, u_{3}, u_{4}\right)$ defined by the formulas

$$
\begin{gathered}
u_{1}=0, \quad u_{2}=\lambda^{2}, \quad u_{3}=\lambda, \\
u_{4}=-\lambda t+\left(l_{2}+l_{3}\right) \beta+\left(m_{2}+m_{3}\right) \gamma \lambda^{3} .
\end{gathered}
$$

If the local coordinates of $R$ be written as $\left(x_{1}, x_{2}, x_{3}, 0\right)$, equations (9) assume the form

$$
\begin{gathered}
u_{1}=0, \quad u_{2}=x_{2} x_{3}^{2}, \quad u_{3}=x_{2}^{2} x_{3}, \\
u_{4}=-x_{1} x_{2} x_{3}+\left(l_{2}+l_{3}\right) \beta x_{2}^{3}+\left(m_{2}+m_{3}\right) \gamma x_{3}^{3}
\end{gathered}
$$

of the most general transformation of Cech [2].

Bell [1] has given a geometric characterization of this general transformation. Lane [7] has characterized this transformation for the special case $l_{2}+l_{3}=m_{2}+m_{3}$.

The quadric $Q_{-\lambda}\left(l_{2}, m_{2}, l_{3}, m_{3}\right)$ induces the transformation

$$
\begin{gathered}
u_{1}=0, \quad u_{2}=x_{2} x_{3}^{2}, \quad u_{3}=x_{2}^{2} x_{3}, \\
u_{4}=-x_{1} x_{2} x_{3}+\left(l_{2}-l_{3}\right) \beta x_{2}^{3}+\left(m_{2}-m_{3}\right) \gamma x_{3}^{3} .
\end{gathered}
$$

The particular quadrics referred to above induce several interesting special transformations (10) and (11).

4. The quadrics $Q_{u}\left(l_{2}, m_{2}, l_{3}, m_{3}\right), Q_{v}\left(l_{2}, m_{2}, l_{3}, m_{3}\right)$. We may characterize some of the quadrics in the family (1) in the following manner. The $S_{\lambda}\left(l_{2}, m_{2}, l_{3}, m_{3}\right)$-associate of the reciprocal of the projective normal joins the points defined by $R_{\lambda}=x_{u}+k_{2} x, S_{\lambda}=x_{v}+k_{3} x, k_{2}, k_{3}$ being defined by (3). The tangent to the locus of $R_{\lambda}$ as $x$ moves along $C_{\lambda}$ and the point $S_{\lambda}$ determine a plane $\pi$. The plane $\pi$ intersects the projective normal in a point $P$. As $x$ moves along $C_{\lambda}$ the plane $x_{3}=0$ envelops a developable surface generated by a line which intersects the projective normal in a point $Q$. The plane determined by $R_{\lambda}, S_{\lambda}$ and the harmonic conjugate of $x$ with respect to $P$ and $Q$ has the equation $T=0, T$ being defined by (2) and wherein 


$$
\begin{aligned}
k_{4}= & \frac{1}{2}\left\{-\frac{\beta}{\lambda^{3}}\left[l_{2} \lambda^{\prime}+\left(l_{2}^{2}+l_{2} l_{3}+l_{3}\right) \beta\right.\right. \\
& \left.+\left[\left(1-l_{2}\right) \frac{\beta_{v}}{\beta}+\theta_{v}\right] \lambda^{2}+l_{2}\left(\theta_{u}-\frac{\beta_{u}}{\beta}\right) \lambda\right] \\
& +m_{2} \gamma\left[2 \lambda^{\prime}-\left(m_{2}+m_{3}\right) \gamma \lambda^{3}+\frac{\gamma_{v}}{\gamma} \lambda^{2}+\left(\frac{\gamma_{u}}{\gamma}-\theta_{u}\right) \lambda\right] \\
& \left.-\theta_{u v}-\left[\left(2 l_{2}+l_{3}\right) m_{2}+m_{3}\left(1+l_{2}\right)+1\right] \beta \gamma\right\} .
\end{aligned}
$$

The quadric having second order contact with $S$ at $x$ and passing through the lines $x_{2}=0, T=0 ; x_{3}=0, T=0$ has the equation (1), $k_{4}$ being given by (12). We shall denote this quadric by $Q_{u}\left(l_{2}, m_{2}\right.$, $\left.l_{3}, m_{3}\right)$.

In an analogous manner we may define a quadric $Q_{v}\left(l_{2}, m_{2}, l_{3}, m_{3}\right)$ with the equation (1) with $k_{4}$ given by the formula

$$
\begin{aligned}
k_{4}= & \frac{1}{2}\left\{-\gamma\left[-m_{3} \lambda^{\prime}+\left(m_{3}^{2}+m_{2} m_{3}+m_{2}\right) \gamma \lambda^{3}\right.\right. \\
& \left.+\left[\left(1-m_{3}\right) \frac{\gamma_{u}}{\gamma}+\theta_{u}\right] \lambda+m_{3}\left(\theta_{v}-\frac{\gamma_{v}}{\gamma}\right) \lambda^{2}\right] \\
& +\frac{l_{3} \beta}{\lambda^{3}}\left[-2 \lambda^{\prime}-\left(l_{2}+l_{3}\right) \beta+\frac{\beta_{u}}{\beta} \lambda+\left(\frac{\beta_{v}}{\beta}-\theta_{v}\right) \lambda^{2}\right] \\
& \left.-\theta_{u v}-\left[\left(2 m_{3}+m_{2}\right) l_{3}+l_{2}\left(1+m_{3}\right)+1\right] \beta \gamma\right\} .
\end{aligned}
$$

5. Applications. It is easily verified that the special quadrics $Q_{u}(-1,0,1,0), Q_{v}(0,1,0,-1)$ are the asymptotic osculating quadrics of the curve $C_{\lambda}$ at $x$. These quadrics may therefore be considered as generalizations of the asymptotic osculating quadrics. The quadric $Q_{u}(0,0,0,0)$ for a curve $C_{\lambda}$ tangent to the asymptotic $u=$ const. (or $Q_{v}(0,0,0,0)$ for a curve $C_{\lambda}$ tangent to $v=$ const. $)$ is the quadric of Lie.

Let us call the quadrics $Q_{u}(0,1,0,-1), Q_{v}(-1,0,1,0)$ the antiasymptotic osculating quadrics. They are given by (1) with the respective values of $k_{4}$ :

$$
\begin{aligned}
k_{4}= & \frac{1}{2}\left\{\gamma\left[2 \lambda^{\prime}+\frac{\gamma_{v}}{\gamma} \lambda^{2}+\left(\frac{\gamma_{u}}{\gamma}-\theta_{u}\right) \lambda\right]\right. \\
& \left.-\frac{\beta}{\lambda}\left(\frac{\beta_{v}}{\beta}+\theta_{v}\right)-\theta_{u v}\right\},
\end{aligned}
$$




$$
\begin{aligned}
k_{4}= & \frac{1}{2}\left\{\frac{\beta}{\lambda^{3}}\left[-2 \lambda^{\prime}+\frac{\beta_{u}}{\beta} \lambda+\left(\frac{\beta_{v}}{\beta}-\theta_{v}\right) \lambda^{2}\right]\right. \\
& \left.-\gamma \lambda\left(\frac{\gamma_{u}}{\gamma}+\theta_{u}\right)-\theta_{u v}\right\} .
\end{aligned}
$$

It is easy to verify the following theorems.

The anti-asymptotic osculating quadrics (assumed distinct) intersect in the asymptotic tangents and in a conic whose plane passes through the projective normal if and only if $C_{\lambda}$ is a pangeodesic. These quadrics coincide if and only if $\beta+\gamma \lambda^{3}=0$, and $\phi+\lambda \psi=0$; that is, the curve $C_{\lambda}$ must be tangent to a curve of Darboux, and that tangent must be the second canonical tangent of $S$ at $x$.

The conjugal quadrics $Q_{u}(0, k, k, 0), Q_{v}(0, k, k, 0)$ have equations given by (1) with the respective values of $k_{4}$ :

$$
\begin{aligned}
k_{4}= & \frac{1}{2}\left\{k \gamma\left[2 \lambda^{\prime}-k \gamma \lambda^{3}+\frac{\gamma_{v}}{\gamma} \lambda^{2}+\left(\frac{\gamma_{u}}{\gamma}-\theta_{u}\right) \lambda\right]\right. \\
& \left.-\frac{\beta}{\lambda^{3}}\left[k \beta+\left(\frac{\beta_{v}}{\beta}+\theta_{v}\right) \lambda^{2}\right]-\theta_{u v}-\left(1+k^{2}\right) \beta \gamma\right\}, \\
k_{4}= & \frac{1}{2}\left\{-k \frac{\beta}{\lambda^{3}}\left[2 \lambda^{\prime}+k \beta-\frac{\beta_{u}}{\beta} \lambda-\left(\frac{\beta_{v}}{\beta}-\theta_{v}\right) \lambda^{2}\right]\right. \\
& \left.-\gamma\left[k \gamma \lambda^{3}+\left(\frac{\gamma_{u}}{\gamma}+\theta_{u}\right) \lambda\right]-\theta_{u v}-\left(1+k^{2}\right) \beta \gamma\right\} .
\end{aligned}
$$

These quadrics coincide if and only if

$$
\begin{aligned}
2 k\left(\beta+\gamma \lambda^{3}\right) \lambda^{\prime}= & k(1-k) \beta^{2}+k \beta_{u} \lambda \\
& +\beta \lambda^{2}\left[(1+k) \frac{\beta_{v}}{\beta}+(1-k) \theta_{v}\right] \\
& -\gamma \lambda^{4}\left[(1+k) \frac{\gamma_{u}}{\gamma}+(1-k) \theta_{u}\right] \\
& -k \gamma_{u} \lambda^{5}-k(1-k) \gamma^{2} \lambda^{6} .
\end{aligned}
$$

The curves represented by (14) are hypergeodesics if and only if $\phi=\psi=0$, that is, $S$ is a coincidence surface. In that case the hypergeodesics represented by (13) are given by

$$
\lambda^{\prime}=\frac{1}{2}(1-k) \beta+\frac{1}{2} \frac{\beta_{u}}{\beta} \lambda-\frac{1}{2} \frac{\gamma_{v}}{\gamma} \lambda^{2}-\frac{1}{2}(1-k) \gamma \lambda^{3} .
$$


The cusp-axis of these hypergeodesics is the projective normal. From (14) we see that the quadrics $Q_{u}(0,1,1,0), Q_{v}(0,1,1,0)$ of Davis coincide if and only if $C_{\lambda}$ is a pan-geodesic. And the curves $C_{\lambda}$ defined by (14) are pan-geodesics if and only if the quadrics are quadrics of Davis.

As the point $x$ moves along $C_{\lambda}$ the asymptotic tangents generate ruled surfaces $R_{u}, R_{v}$. Hsiung [6] has shown the existence of a pair of quadrics associated with $R_{u}$ and $R_{v}$. He cuts these surfaces by a plane through the points $P_{u}=x_{u}+A x, P_{v}=x_{v}+B x$. The locus of the conic having ordinary contact at $P_{u}$ and second order contact at $P_{v}$ with these sections is a quadric $Q(-1,0,0,-1)$ whose equation is (1) with $k_{4}$, given by the formula

$$
\begin{aligned}
k_{4}= & \frac{1}{2}\left\{\frac{\beta}{\lambda^{3}}\left[\lambda^{\prime}-\lambda\left(\theta_{u}-\theta_{v} \lambda-\phi+2 \psi \lambda\right)\right]+\frac{\beta^{2}}{\lambda^{3}}\right. \\
& \left.+2 A \lambda\left(\beta+\gamma \lambda^{3}\right)+\beta \gamma-\theta_{u v}\right\} .
\end{aligned}
$$

This quadric of Hsiung coincides with $Q_{u}(-1,0,0,-1)$ if and only if $P_{u}$ is the point whose coordinates are given by the expression

$$
P_{u}=x_{u}-\beta x / \lambda .
$$

Interchanging the roles of the asymptotic curves and ruled surfaces, a second point $P_{v}$ with coordinates

$$
P_{v}=x_{v}-\gamma \lambda x
$$

is characterized.

The $R_{\lambda}$-associate of the reciprocal of the projective normal, that reciprocal, the line $P_{u} P_{v}$ defined by (15) and (16), and the tangent to the $R_{\lambda-}$ derived curve through $x$ are concurrent, and are moreover harmonic. The $R_{\lambda}$-associate of the line $P_{u} P_{v}$ is the projective normal.

It is known [7] that the asymptotic osculating quadric $Q_{v}(0,1,0,-1)$ reduces to the quadric of Wilczynski if $C_{\lambda}$ is tangent to the asymptotic curve $v=$ const., and has an inflexion at $x$, and to the quadric of Fubini if $C_{\lambda}$ is tangent to $v=$ const., and has the tangent plane to $S$ at $x$ as stationary osculating plane. These quadrics are respectively the special quadrics $h=1, h=1 / 3$ of the pencil

$$
x_{2} x_{3}+x_{4}\left\{-x_{1}-(1 / 2)\left[\theta_{u v}+(1-h) \beta \gamma\right] x_{4}\right\}=0 .
$$

Lane [8] has characterized the invariant parameter $h$ of the pencil (17) in terms of a cross ratio whose elements involve the quadric of Lie $(h=0)$ and the quadric of Wilczynski $(h=1)$. The definition (8) of $m_{3}$ enables us to describe the invariant parameter $h$ without recourse to any special quadric of (17). 
The quadric $Q_{v}\left(0, m_{2}, 0, m_{3}\right)$ for a curve $C_{\lambda}$ tangent to $v=$ const. and having an inflexion at $x\left(\lambda=0, \lambda^{\prime}+\beta=0\right)$ has the equation (17) with $h=-m_{3}$. The definitions (8) imply that $l=1, k=0, m_{3}=L$, $m_{2}=K$. From (6) we find that $R=\rho_{\mu}, S=\sigma_{\lambda}$. Then the points $R_{\lambda}, S_{\lambda}$ determining the $S_{\lambda}\left(0, m_{2}, 0, m_{3}\right)$-associate of $l$ are found from the cross ratio equations

$$
\left(x, \rho_{\mu}, \rho, R_{\lambda}\right)=K, \quad\left(x, \sigma_{\lambda}, \sigma, S_{\lambda}\right)=L=-h .
$$

\section{REFERENCES}

1. P. O. Bell, $A$ study of curved surfaces by means of certain associated ruled surfaces, Trans. Amer. Math. Soc. vol. 46 (1939) pp. 389-409.

2. E. Čech, $L$ ' intorno d'un punto d'una superficie considerato dal punto di vista proiettiva, Annali di Matematica (3) vol. 31 (1922) pp. 191-206.

3. W. M. Davis, Contributions to the general theory of conjugate nets, Dissertation, Chicago, 1932. 564.

4. L. Green, The axial quadrics of a surface, Duke Math. J. vol. 10 (1943) pp. 557-

5. V. G. Grove, The transformation of Čech, Bull. Amer. Math. Soc. vol. 50 (1944) pp. 231-234.

6. C. C. Hsiung, Plane sections of certain ruled surfaces associated with a curved surface, Duke Math. J. vol. 11 (1944) pp. 59-64.

7. E. P. Lane, A treatise on projective differential geometry, The University of Chicago Press, 1942.

8. - The correspondence between the tangent plane of a surface and its point of contact, Amer. J. Math. vol. 48 (1926) pp. 204-214.

9. George Wu, Systems of quadrics associated with a point on a surface, I, II, Duke Math. J. vol. 10 (1943) pp. 499-513, 515-530.

Michigan State College 